\section{RMD Open}

Rheumatic \& Musculoskeletal Diseases

\title{
Clinical manifestations and outcome of
} SARS-CoV-2 infections in children and adolescents with rheumatic musculoskeletal diseases: data from the National Paediatric Rheumatology Database in Germany

\begin{abstract}
Claudia Sengler (D) , ${ }^{1}$ Sascha Eulert, ${ }^{1}$ Kirsten Minden, ${ }^{1,2}$ Martina Niewerth, ${ }^{1}$ Gerd Horneff,, ${ }^{3,4}$ Jasmin Kuemmerle-Deschner, ${ }^{5}$ Caroline Siemer, ${ }^{6}$ Rainer Berendes, ${ }^{7}$ Hermann Girschick, ${ }^{8}$ Regina Hühn, ${ }^{9}$ Michael Borte, ${ }^{10}$ Anton Hospach, ${ }^{11}$ Wolfgang Emminger, ${ }^{12}$ Jakob Armann, ${ }^{13}$ Ariane Klein, ${ }^{3,4}$ Tilmann Kallinich ${ }^{2}$
\end{abstract}

To cite: Sengler C, Eulert S, Minden $\mathrm{K}$, et al. Clinical manifestations and outcome of SARS-CoV-2 infections in children and adolescents with rheumatic musculoskeletal diseases: data from the National Paediatric Rheumatology Database in Germany. RMD Open 2021;7:e001687. doi:10.1136/ rmdopen-2021-001687

- Additional supplemental material is published online only. To view, please visit the journal online (http://dx.doi.org/10. 1136/rmdopen-2021-001687).

CS and SE are joint first authors. $\mathrm{AK}$ and $\mathrm{TK}$ are joint senior authors.

Received 1 April 2021 Accepted 16 July 2021

Check for updates

(C) Author(s) (or their employer(s)) 2021. Re-use permitted under CC BY-NC. No commercial re-use. See rights and permissions. Published by BMJ.

For numbered affiliations see end of article.

Correspondence to

Claudia Sengler; sengler@drfz.de

\section{ABSTRACT}

Objectives This study aimed to investigate the clinical manifestations, course and outcome of SARSCoV-2 infection among children and adolescents with rheumatic and musculoskeletal diseases (RMD). Due to their underlying disease as well due to therapeutic immunosuppression, these patients may be at risk for a severe course of COVID-19 or for a flare of the underlying disease triggered by SARS-CoV-2 infection.

Methods Demographic, clinical and treatment data from juvenile patients with RMD as well as data about SARS-CoV-2 infection like test date and method, clinical characteristics, disease course, outcome and impact on the disease activity of the RMD were documented on a specific SARS-CoV-2 questionnaire implemented in the National Paediatric Rheumatology Database (NPRD) in Germany. The survey data were analysed descriptively. Results From 17 April 2020 to 16 February 2021, data were collected from 76 patients (52\% female) with RMD and laboratory-proven SARS-CoV-2 infection with median age of 14 years, diagnosed with juvenile idiopathic arthritis (58\%), autoinflammatory (24\%) and connective tissue disease (8\%). Fifty-eight patients $(76 \%)$ received diseasemodifying antirheumatic drugs (DMARDs), $41 \%$ biological DMARDs and $11 \%$ systemic glucocorticoids. Fifty-eight (76\%) had symptoms of COVID-19. Disease course of SARS-CoV-2 infection (classified as asymptomatic, mild, moderate, severe, life-threatening) was mild and outcome of COVID-19 (classified as recovered, not yet recovered, permanent damage or deceased) was good (recovered) in the majority of patients. Two patients were hospitalised, one of whom required intensive care and died of cardiorespiratory failure. In $84 \%$ of SARS-CoV-2-positive patients, no relevant increase in disease activity of the RMD was observed.

Conclusions In our cohort, SARS-CoV-2 infection in juvenile patients with RMD under various medications was

\section{Key messages}

What is already known about this subject?

- Children and adolescents develop COVID-19 less frequently and usually more mildly than adults.

- The clinical characteristics of SARS-CoV-2 infection in respect to symptoms, severity of the course and outcome as well as the influence on the underlying disease in juvenile patients with rheumatic musculoskeletal diseases (RMD) under different therapeutic regimens are unclear so far.

What does this study add?

- In the study presented here, the clinical characteristics and outcome of SARS-CoV-2 infection in juvenile patients with RMD were explored using a survey within the National Paediatric Rheumatology Database.

- The clinical course of SARS-CoV-2 infection in patients with rheumatic diseases was mild in most cases, regardless of whether immunosuppressive therapy was maintained or discontinued.

- SARS-CoV-2 infection (symptomatic or asymptomatic) does not appear to have a major effect on the underlying disease activity.

mild with good outcome in the majority of cases and does not appear to have a relevant impact on disease activity of the underlying condition.

\section{INTRODUCTION}

Children and adolescents seem to be less affected by COVID-19 and to have a milder 


\section{Key messages}

How might this impact on clinical practice or future developments?

- It may be concluded that continuation of immunosuppressive therapy in juvenile patients with RMD with evidence of SARS-CoV-2 infection does not pose a risk for a more severe course of COVID-19.

- Further studies with larger case numbers are needed to better investigate associations between a distinct underlying disease, its specific drug therapy, and the clinical expression and possible consequences of SARS-CoV-2 infection in these patients.

course of COVID-19 than adults. ${ }^{1}$ However, similar to adults, a pre-existing underlying disease or immunosuppression is described as a risk factor for hospitalisation in children. ${ }^{2}$ Children and adolescents with rheumatic musculoskeletal disorders (RMD) may be particularly susceptible to SARS-CoV-2 infection, partly because of their underlying disease and also because of therapeutically induced immunosuppression. Furthermore, the immunosuppressive therapy may influence the clinical course of SARS-CoV-2 infection positively or negatively, also SARS-CoV-2 infection might alter the disease activity of the underlying chronic inflammatory disease.

To address these issues, a specific SARS-CoV-2 questionnaire was implemented into the National Paediatric Rheumatology Database (NPRD) in Germany in 2020 to prospectively gather data (i) on clinical characteristics, treatment and outcome of SARS-CoV-2 infection and (ii) on the impact of SARS-CoV-2 infection on the preexisting conditions in juvenile patients with RMD under different immunosuppressive therapies.

Here, we report the demographic and clinical characteristics of 76 children and adolescents with RMD and confirmed SARS-CoV-2 infection.

\section{PATIENTS AND METHODS}

\section{National Paediatric Rheumatology Database}

The NPRD started $1997^{3}$ and is now being conducted at $>60$ different paediatric rheumatology sites throughout Germany. Yearly, about 15000 children and adolescents with different RMD, most commonly with juvenile idiopathic arthritis (JIA), and with connective tissue diseases (eg, juvenile systemic lupus erythematosus, juvenile dermatomyositis (jDM)), vasculitis or autoinflammatory diseases are recorded in the NPRD in a standardised format by disease-specific questionnaires.

The SARS-CoV-2 module of the NPRD was set up on recommendation of the Society for Paediatric Rheumatology (GKJR) in Germany to provide reliable and representative epidemiological data on the frequency, course and outcome of SARS-CoV-2 infection in paediatric patients with RMD. This SARS-CoV-2 module is embedded as a cross-sectional study in the established prospective NPRD and collects information from patients with RMD and all SARS-CoV-2 infection courses (also including asymptomatic SARS-CoV-2 infection), by means of a specific paper-based questionnaire.

The following items were recorded: demographic parameter, information on SARS-CoV-2 testing (method, reason), symptoms and clinical course of SARS-CoV-2 infection, outcome of COVID-19, treatment of the SARS-CoV-2 infection as well as of the underlying disease. In addition, disease activity of the rheumatic disease (21-item numeric rating scale (NRS 0-10, 0: best)) was recorded before and after SARS-CoV-2 infection.

This questionnaire had been previously aligned with those of the EULAR COVID-19 Registry ${ }^{4}$ and the COVID-19 Global Rheumatology Alliance Registry. ${ }^{5}$ The introduction of the SARS-CoV-2 module into the NPRD was communicated to all members via email through the GKJR on 16 April 2020, and reporting of appropriate patients was also encouraged through this channel.

\section{Biologics in Paediatric Rheumatology Registry}

This data collection was supplemented by cases of SARSCoV-2-positive children and adolescents with RMD from the Biologics in Paediatric Rheumatologiy Registry (BiKeR) ${ }^{6}{ }^{6}$ in which SARS-CoV-2 infection was recorded as an 'event of special interest'.

\section{German Society for Paediatric Infectiology}

In order to cover the spectrum of SARS-CoV-2 infection in children and adolescents with rheumatic diseases as completely as possible, additional cases were reported to us via the COVID-19 Survey of the German Society for Paediatric Infectiology (DGPI, https://dgpi.de/), a registry of hospitalised children and adolescents with SARS-CoV-2 infection, who were identified by indicating a corresponding comorbidity.

A detailed description of the data collection in the respective registries can be found in the online supplemental material.

Only patients with laboratory evidence of SARS-CoV-2 infection were included in our analysis. Data collection for the manuscript was completed before the introduction of systematic rapid antigen-based tests for SARS-CoV-2 screening in schools. As in the rest of the population, children and adolescents with RMD were generally only tested for the presence of SARS-CoV-2 infection if they had contact with an infected person or if they had symptoms during the period in which the data for this manuscript were collected (April 2020, first wave, until February 2021, phase-out of the second wave). The indication for testing was documented on the questionnaire.

\section{Statistics}

Statistical analyses were performed using IBM SPSS Statistics V.26 and SAS V.9.4M5. Plots were made with Microsoft Excel 2016. Results for continuous variables 
are presented as medians and IQRs unless otherwise stated. For categorical variables, absolute count is given and proportions of corresponding categories are calculated and presented as percentages. Missing responses were treated on a per-category basis and did not lead to an exclusion of the corresponding case. The difference in the NRS ( $\triangle \mathrm{NRS})$, which was used to describe the change of the underlying RMD disease activity, was calculated as the difference of the NRS of the outcome date (NRS-out) and the pre-infection NRS (NRS-pre). Consequently, a non-positive difference describes a constant/ decrease of disease activity while a positive difference implies an increase in disease activity. Change in disease activity (' $\Delta$ NRS=NRS-out-NRS-pre') was further categorised as follows: for increase: minimal, if $0<\Delta \mathrm{NRS} \leq 1$; minor, if $1<\Delta \mathrm{NRS} \leq 2$; relevant if $\Delta \mathrm{NRS}>2$. For decrease: minimal/no decrease: $-1 \leq \Delta \mathrm{NRS} \leq 0$; minor decrease: -2 $\leq \Delta$ NRS $<-1$; relevant decrease: $\Delta$ NRS $<-2$. This item was evaluated only in individuals with a date of COVID-19 symptom onset, or-if asymptomatic - with a date of PCR or antigen testing, because these methods allowed to determine the point of time the disease/infection occurred (in contrast to antibody testing).

To determine whether the NRS values were reported in chronological order, that is, the NRS timeline can be clearly separated into before/after SARS-CoV-2 infection, only valid dates were considered. In cases where only the day was missing, we imputed the missing value with the 15 th of the corresponding month.

\section{RESULTS}

\section{Demographic parameters, diagnostics and treatment}

From 17 April 2020 until 16 February 2021, data of 76 patients with RMD were collected. They were predominantly adolescent, and boys and girls were documented with equal frequency. The majority of patients suffered from JIA (58\%), $24 \%$ of patients were diagnosed with an autoinflammatory disease (table 1). In addition to the underlying RMD, five patients suffered from asthma, four from uveitis, two from allergies and one patient each from ventricular septal defect, aortic valve insufficiency, Hashimoto's thyroiditis, hypothyroidism and diabetes mellitus.

Most patients were tested for SARS-CoV-2 because they had contact with an infected person $(50,66 \%)$; only $25(33 \%)$ were tested because of typical symptoms. The preferred test method was PCR $(71 \%)$, followed by serology testing (25\%) and antigen detection (4\%).

Fifty-eight $(76 \%)$ were treated with a DMARD at the time of SARS-CoV-2 infection, and $41 \%$ with a biological DMARD (bDMARD). DMARD therapy $(9 \times$ bDMARD: etanercept, adalimumab, golimumab, tocilizumab, $2 \times$ bDMARD+methotrexate (MTX), 3× MTX only) was discontinued or changed in frequency of application and/ or dosage (the entire therapy in each case) in response to confirmed SARS-CoV-2 infection in 14 patients with the following diagnoses: seronegative polyarthritis $(\mathrm{PA}) \mathrm{n}=7$, enthesitis-related arthritis $n=2$, seropositive PA, oligoarthritis (OA) persistent, OA extended, chronic recurrent multifocal osteomyelitis, deficiency of adenosine deaminase 2 each $n=1$.

\section{Clinical characteristics of SARS-CoV-2 infection in juvenile patients with RMD}

Fifty-eight (76\%) developed symptoms of COVID-19, 18 patients (24\%) remained asymptomatic.

All patients with autoinflammatory diseases $(n=18)$ as well as all patients with systemic JIA-even though only three patients-developed symptoms of COVID-19 whereas $27 \%$ of patients with the other forms of JIA and $50 \%$ of patients with connective tissue diseases remained asymptomatic.

The clinical symptoms of SARS-CoV-2 infection in the 58 symptomatic juvenile patients with RMD are summarised in figure 1 . The most common symptoms were rhinitis and fever (40\%), although usually not high: of the 13 patients who provided information on fever level, only 1 had a body temperature $>39.5^{\circ} \mathrm{C} ; 6$ patients reported a temperature in the range of $38.5^{\circ} \mathrm{C}$ and $39.5^{\circ} \mathrm{C}$ and 6 patients reported an elevated temperature $<38.5^{\circ} \mathrm{C}$.

One patient who was hospitalised was diagnosed with pneumonia, but no outpatients $(n=74)$ were reported to have pneumonia; two outpatients were diagnosed with bronchitis/bronchiolitis.

Due to the small number of documented laboratory results, we were unable to perform any analyses in this regard.

\section{Course and outcome of COVID-19 in juvenile patients with RMD}

Disease course was classified as mild in 44 of 46 symptomatic patients $(96 \%)$ with valid data on this item. None of the symptomatic patients with missing data regarding the disease course of COVID-19 ( $n=12)$ were hospitalised.

Two patients were reported via the DGPI registry for hospitalised children with COVID-19: a girl with chronic non-bacterial osteitis on therapy with NSAID was hospitalised but did not show clinical signs of pneumonia nor required oxygen administration or intensified drug therapy. The second patient was a girl who was initially classified as having systemic JIA at the age of 1 year and then as polyarticular JIA in the further disease course, and treated with MTX as well as prednisolone. After having experienced a second febrile seizure she was diagnosed with sinus vein thrombosis and cerebral oedema on CT. This patient died despite intensive care 8 days after symptom onset. The cause of death was considered cardiorespiratory failure with radiographic changes in chest X-ray compatible with COVID-19. A genetic examination revealed a so far unrecognised immunodeficiency.

Of the 51 symptomatic patients for whom COVID-19 outcome information was available, 40 patients (78\%) reported that their health status was recovered (median time from symptom onset to outcome evaluation 28 days (IQR 9-55), 10 patients (20\%) stated that their health 
Table 1 Demographic parameter, diagnostic tests, diagnoses and treatment of 76 juvenile patients with RMD with laboratory-proven SARS-CoV-2 infection

\begin{tabular}{|c|c|c|c|}
\hline Patients & Total cohort & Symptomatic & Asymptomatic \\
\hline Total & 76 & 58 & 18 \\
\hline Female, \% & 52 & 55 & 44 \\
\hline Age, median (IQR) & $14(11-16.5)$ & $14(12-17)$ & $12(7-16)$ \\
\hline \multicolumn{4}{|l|}{ Test method, n (\%) } \\
\hline PCR & $54(71)$ & $41(71)$ & $13(72)$ \\
\hline Antigen test & $3(4)$ & $3(5)$ & $0(0)$ \\
\hline Antibody test & $19(25)$ & $14(24)$ & $5(28)$ \\
\hline Juvenile idiopathic arthritis, $\mathrm{n}(\%)$ & $44(58)$ & $33(57)$ & $11(61)$ \\
\hline Oligoarthritis (persistent/extended) & 16 & 11 & 5 \\
\hline Polyarthritis (RF negative/positive) & 17 & 15 & 2 \\
\hline Enthesitis-related arthritis & 7 & 4 & 3 \\
\hline Systemic juvenile idiopathic arthritis & 3 & 3 & 0 \\
\hline Other arthritis & 1 & 0 & 1 \\
\hline Autoinflammatory diseases, $\mathrm{n}(\%)$ & $18(24)$ & $18(31)$ & 0 \\
\hline FMF & 11 & 11 & 0 \\
\hline NLRP3-associated autoinflammatory disease & 2 & 2 & 0 \\
\hline PFAPA & 1 & 1 & 0 \\
\hline TRAPS & 3 & 3 & 0 \\
\hline DADA2 & 1 & 1 & 0 \\
\hline Connective tissue disease, $\mathrm{n}(\%)$ & $6(8)$ & $3(5)$ & $3(17)$ \\
\hline SLE & 3 & 2 & 1 \\
\hline JDM & 1 & 0 & 1 \\
\hline Sjögren's syndrome & 1 & 1 & 1 \\
\hline Scleroderma (localised) & 1 & 0 & 1 \\
\hline Other, $\mathrm{n}(\%)$ & $8(11)$ & $4(7)$ & $4(22)$ \\
\hline Behçet's disease & 1 & 1 & 0 \\
\hline CRMO & 4 & 2 & 2 \\
\hline $\mathrm{SpA}^{*}$ & 1 & 1 & 0 \\
\hline Sjögren's syndrome & 1 & 1 & 0 \\
\hline Uveitis (idiopathic) & 2 & 0 & 2 \\
\hline Treatment, $\mathrm{n}(\%)$ & $66(87)$ & $49(84)$ & $17(94)$ \\
\hline Systemic glucocorticoids & 8 & 4 & 4 \\
\hline Any DMARD & 58 & 49 & 9 \\
\hline MTX & 18 & 13 & 5 \\
\hline bDMARD & 31 & 27 & 4 \\
\hline TNF- $\alpha$ antagonist & 19 & 17 & 2 \\
\hline MTX+TNF- $\alpha$ antagonist & 4 & 4 & 0 \\
\hline IL-6 blockade & 6 & 5 & 1 \\
\hline IL-1 blockade & 4 & 4 & 0 \\
\hline Baricitinib & 1 & 1 & 0 \\
\hline Colchicine & 9 & 9 & 0 \\
\hline Hydroxychloroquine & 7 & 4 & 3 \\
\hline Mycophenolate mofetil & 3 & 2 & 1 \\
\hline Sulfasalazine & 1 & 1 & 0 \\
\hline Azathioprine & 2 & 2 & 0 \\
\hline
\end{tabular}

(b)DMARD, (biological) disease-modifying antirheumatic drug; CRMO, chronic recurrent multifocal osteomyelitis; DADA2, deficiency of adenosine deaminase 2; FMF, familial Mediterranean fever ; IL, interleukin; JDM, juvenile dermatomyositis; MTX, methotrexate; PFAPA, periodic fever, aphthous stomatitis, pharyngitis, cervical adenitis; RF, rheumatoid factor; SLE, systemic lupus erythematosus; SpA, spondylarthritis, the patient was older than 16 years at the time of diagnosis and therefore could not be classified as having enthesitis-related arthritis; TNF, tumour necrosis factor; TRAPS, TNF-receptor-associated periodic syndrome. 


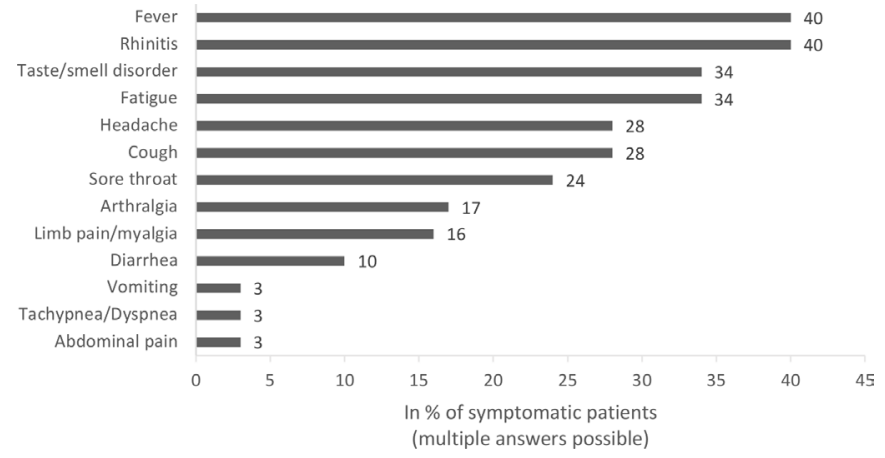

Figure 1 Clinical symptoms of SARS-CoV-2 infection in 58 juvenile patients with rheumatic and musculoskeletal diseases (RMD).

status had not yet recovered after COVID-19, mostly due to a continuing taste/smell disorder (median time between symptom onset and outcome evaluation was 26 days, IQR 7-47).

\section{Outcome of the underlying RMD after SARS-CoV-2 infection}

We were able to evaluate the disease activity of the underlying RMD (NRS) before and after the proven SARS-CoV-2 infection in 32 individuals (28 symptomatic and 4 asymptomatic patients). The remaining patients in our study lacked the necessary information to do so. The median time from the last assessment of RMD disease activity of the chronic inflammatory disease to positive SARS-CoV-2 testing was 52 days (IQR 37.5102.5 days). After detection of SARS-CoV-2 infection, the median time to outcome assessment of RMD disease activity was 31 days (IQR $13-50$ days). The mean $( \pm \mathrm{SD})$ disease activity with a range of 0 (=best) to 10 of those 32 patients was $0.90 \pm 1.37$ (median 0.5, IQR 1-1.25) before SARS-CoV-2 infection and 1.14 \pm 2.12 (median 0.25, IQR $0-1.25)$ at the time of outcome assessment. No up to minimal increase in disease activity (difference in NRS before vs after SARS-CoV-2 infection $\leq 1$ ) of the underlying disease was recorded in 27 of 32 patients (84\%), regardless of whether patients were asymptomatically infected or developed COVID-19 (figure 2). An increase in disease activity with a difference in NRS before versus after SARS-CoV-2 infection $>1$ was seen in five patients (four symptomatic, one asymptomatic).

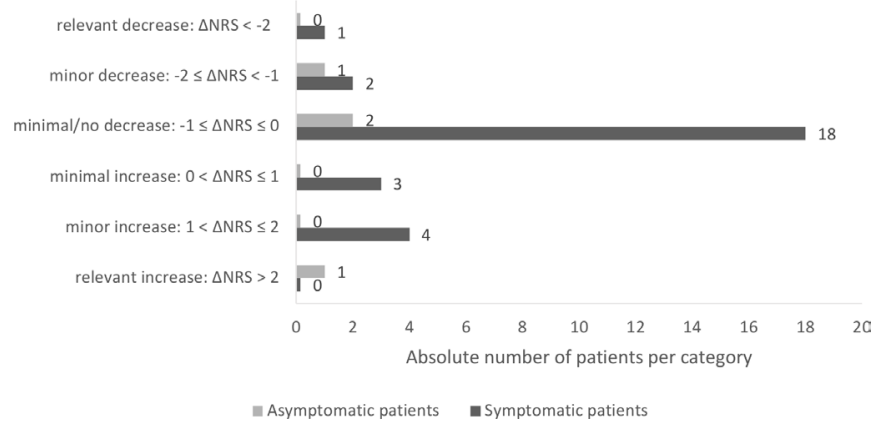

Figure 2 Changes in numeric rating scale (NRS) disease activity of the underlying rheumatic disease before versus after SARS-CoV-2 infection.
All but one $(n=13)$ of the patients in whom drug therapy had been discontinued or the dosing/interval of application of medication had been changed had an NRS of $\leq 1$ with respect to disease activity of the underlying disease before SARS-CoV-2 infection, which changed by a maximum of 0.5 thereafter. One patient, who had paused tocilizumab for two doses, experienced a flare of his seronegative PA (NRS-out: 9) 2 months after asymptomatic SARS-CoV-2 infection (NRS before infection was 3).

Another patient experienced a recurrence of uveitis 8 weeks after symptom onset of COVID-19 without a flare of her OA while maintaining DMARD therapy with MTX.

\section{DISCUSSION}

Although children and adolescents are known to develop COVID-19 less frequently and usually more mildly than adults, ${ }^{178}$ the clinical characteristics of a SARS-CoV-2 infection in terms of symptoms, severity of course and outcome as well as the impact of this infection on the underlying disease in juvenile patients with rheumatic or autoinflammatory diseases, has remained unclear.

In the study presented here, the majority of patients diagnosed with RMD under different therapeutic regimens with confirmed SARS-CoV-2 infection have shown a mild disease course with good outcome. Two patients were hospitalised, of whom one patient had a severe course of COVID-19 with a fatal outcome. To our knowledge, our cohort currently represents the largest collection of data from children and adolescents with RMD and confirmed SARS-CoV-2 infection on various immunomodulatory therapies.

Of the total 76 patients in our cohort, $24 \%$ were asymptomatically infected and $76 \%$ showed symptoms of illness from COVID-19. Fever and rhinitis were most commonly reported with each $40 \%$, followed by fatigue, loss of smell and/or taste $(34 \%)$, headache, cough and sore throat. The frequency distribution of symptoms found here is largely consistent with published data on the clinical manifestation of COVID-19 in children and adolescents in the general population. ${ }^{189}$ However, we have a relatively high proportion of SARS-CoV-2-positive patients in our cohort who reported smell and/or taste disturbances, which has been identified as a fairly specific sign of SARS-CoV-2 infection. ${ }^{10}$

In our cohort, the disease course of COVID-19 was mild in $96 \%$ of patients with valid data for this item; none of the patients who lacked formal information on COVID-19 disease course was hospitalised, so it may be concluded that these patients at least did not have a severe course of COVID-19. Due to this rather homogeneous result in the evaluation of COVID-19 disease course and the low number of hospitalised patients, the influence of comorbidities or drug therapy of the underlying disease on the expression of SARS-CoV-2 infection could not be analysed. From the National Registry of Adult Patients with Rheumatic Diseases and SARS-CoV-2 Infection ${ }^{11}$ in Germany, it was reported that glucocorticoid therapy but 
not DMARD therapy was associated with a higher risk of hospitalisation related to COVID-19. DMARDs may even prevent a severe COVID-19 course, as they also attenuate immune processes triggered by the viral disease, including an exaggerated immune response such as the so-called 'cytokine storm'. Studies on the efficacy of antirheumatic therapeutics in severe COVID-19 courses showed partly conflicting, ${ }^{12-15}$ partly promising results. ${ }^{16}$

Koker et $a l^{17}$ surveyed parents of 414 children and adolescents with rheumatic diseases and immunosuppressive treatment by telephone interview regarding possible COVID-19-associated symptoms, risk contacts, performance of diagnostic tests (SARS-CoV-2-PCR) and possible interruption of antirheumatic therapy, and recorded clinical parameters and therapies from medical records. At the time of the survey, $42.3 \%$ of patients were receiving bDMARDs, $28.7 \%$ non-biological DMARDs and $14.5 \%$ both; a quarter of the cohort had comorbidities. Nine patients were screened in hospital for COVID-19, of whom six had close contact with a confirmed infectious case; only one patient was found to have SARS-CoV-2 infection (with an uncomplicated course).

Some studies investigated the rate of hospitalisationas a surrogate parameter for a more severe course of COVID-19-in adult patients with rheumatic diseases compared with the normal population: Haberman $e t a l^{18}$ reported a similar rate of hospitalisation of patients with RMD in the setting of SARS-CoV-2 infection compared with the normal population in New York City. Jovani et $a l^{19}$ reported an estimated OR of 2.61 for hospitalisation of patients with RMD on bDMARD/targeted synthetic DMARD but found no elevated risk of developing severe COVID-19 in these patients compared with healthy subjects. Nevertheless, some studies actually showed evidence of a more severe course of COVID-19 in patients with rheumatic diseases: D'Silva $e t a t^{20}$ compared manifestation and outcome of COVID-19 in patients with and without RMD and found similar symptoms and laboratory changes as well as comparable hospitalisation and mortality rates in both patient groups. However, patients with RMD were more likely to need intensive care and also mechanical ventilation (multivariable OR 3.11 with $95 \%$ CI 1.07 to 9.05$).{ }^{20}$ Recently, using registry data from Denmark, Cordtz et $a l^{21}$ reported that adult patients with inflammatory rheumatic diseases were both more frequently hospitalised and at higher risk of severe COVID-19 (as measured by the number of patients with intensive therapy, acute respiratory distress syndrome or death). Differences in the composition of the patient populations with respect to age, underlying disease (rheumatoid arthritis, ankylosing spondylitis, connective tissue disease, vasculitis, systemic autoimmune disease, etc), disease duration and comorbidities as well as therapies might explain these discrepant findings.

In our study with juvenile patients with RMD, $96 \%$ of symptomatically SARS-CoV-2-infected individuals had a mild disease and the overall outcome of COVID-19 in these patients with defined chronic rheumatic or autoinflammatory diseases was also good with health condition restored in $78 \%$ of patients 4 weeks after symptom onset of COVID-19. None of the outpatients was diagnosed with pneumonia, two patients were hospitalised. One patient died in whom next-generation sequencing revealed a so far unrecognised underlying immunodeficiency that was presumably responsible for the fatal course, so this patient did not have a 'classic' JIA.

Due to some partially incompletely filled-out questionnaires, for the NRS before and after SARS-CoV-2 infection only limited data sets were available. In $84 \%$ of our patients, who could be evaluated for this item $(n=32)$, there was no or only minimal increase in disease activity of the underlying RMD (increase in NRS $\leq 1$ ). One patient with comorbid anterior uveitis suffered a uveitis recurrence after COVID-19 without a flare of her OA while maintaining DMARD therapy with MTX. In contrast, one patient with seronegative PA experienced a flare of his PA after discontinuation of tocilizumab in the setting of asymptomatic SARS-CoV-2 infection. This patient, unlike all other patients who had DMARD therapy reduced or discontinued after diagnosis of SARS-CoV-2 infection with no increase of disease activity thereafter, had an NRS of 3 (moderate disease activity) already prior to his SARS-CoV-2 infection. Here, the pause in therapy rather than the SARS-CoV-2 infection (especially as asymptomatic) seems to be causative for the flare, particularly since the patient was not in remission before this treatment was stopped.

\section{Limitations}

We started data collection using the SARS-CoV-2 questionnaire of the NPRD immediately after its consensus by the board of the GKJR in April 2020 (during the first wave) and chose a data cut-off date in mid-February 2021 for the manuscript, at the end of the second wave. In Germany, approximately 385000 children and adolescents under 20 years of age had been diagnosed with SARS-CoV-2 infection by the end of March 2021. Of these, 4789 patients were hospitalised $(1.2 \%)$, and 68 required intensive care $(1.4 \%$ of hospitalised juvenile patients). In addition, 11 deaths $(0.22 \%$ of hospitalised children and adolescents, $0.0028 \%$ of the total cohort) were reported in association with SARS-CoV-2 infection, of these, 8 cases had pre-existing conditions. ${ }^{22}$ This survey is a collection of spontaneous registrations. Therefore, we can neither assume to have recorded all cases of SARS-CoV-2-positive children and adolescents with RMD nor to have established a representative group. Thus, due to limited number of cases, neither a valid hospitalisation rate nor a mortality rate of juvenile patients with RMD can be derived from our cohort. We are also unable to draw conclusions about the incidence and/or prevalence of SARS-CoV-2 infection in children and adolescents with rheumatic diseases. The relatively small number of cases of each disease entity and the variety of therapies do not allow reliable conclusions regarding implications of a specific rheumatic disease or the effects of medication 
on the clinical expression and outcome of SARS-CoV-2 infection in these patients. Assessment of disease activity before and after SARS-CoV-2 infection was performed as part of routine clinical practice and not after a fixed interval. Therefore, it is possible that flares that occurred in the period after SARS-CoV-2 infection but before outcome assessment were not recorded. Furthermore, we did not have a comparison group of SARS-CoV-2negative patients with RMD regarding the change in NRS of disease activity over a comparable period of time.

Using data from the BiKeR, it was shown that both biologics therapy and disease activity of JIA are risk factors for severe infections in children and adolescents with JIA. ${ }^{23}$ Therefore, there was concern that SARS-CoV-2 infection might be more common and severe in these patients than was the case in otherwise healthy children. Since this was a novel infectious agent, only assumptions could be made in this regard, as data and experience with it were initially lacking. In addition to the call for documentation of children and adolescents with rheumatic diseases and proven SARS-CoV-2 infection in the NPRD, which was communicated by the German Society for Paediatric and Adolescent Rheumatology in April 2020 , the topic was again brought to the attention of all members in May 2020, when they were contacted by email and asked to participate in an online survey to obtain an opinion on DMARDs therapy in children and adolescents with rheumatic diseases affected by COVID19. ${ }^{24}$ Therefore, we may assume that there was a high level of attention regarding the recording of SARS-CoV-2 infections in these patients. In particular, severe courses usually receive higher attention and would accordingly tend to be documented more frequently (reporting bias); mild courses are more likely to be under-reported.

\section{Summary}

The clinical course of SARS-CoV-2 infection in patients with rheumatic diseases was mild in most juvenile patients with RMD, regardless of whether immunosuppressive therapy was maintained or discontinued. Furthermore, SARS-CoV-2 infection did not appear to have a major effect on the underlying disease activity, whereas discontinuation of therapy seemed to pose a risk of flare in patients with (moderately) active disease.

Further studies with larger numbers of cases are needed to better investigate associations between the underlying disease, its drug therapy and the clinical expression and possible consequences of a SARS-CoV-2 infection in these patients.

\footnotetext{
Author affiliations

${ }^{1}$ Epidemiology Unit, German Rheumatism Research Center Berlin, Berlin, Germany ${ }^{2}$ Department of Pediatric Respiratory Medicine, Immunology and Critical Care Medicine, Charité Universitätsmedizin Berlin, Berlin, Germany

${ }^{3}$ General Paediatrics, Asklepios Clinic Sankt Augustin, Sankt Augustin, Germany ${ }^{4}$ Department of Paediatrics and Adolescent Medicine, University Hospital Cologne, Cologne, Germany

${ }^{5}$ Department of Paediatrics, University Hospital Tübingen, Tübingen, Germany

${ }^{6}$ German Center for Paediatric and Adolescent Rheumatology, Garmisch-

Partenkirchen, Germany
}

${ }^{7}$ Department of Paediatric Rheumatology, Kinderkrankenhaus Sankt Marien gGmbH, Landshut, Germany

${ }^{8}$ Department of Paediatrics, Vivantes Clinic in Friedrichshain, Berlin, Germany ${ }^{9}$ Clinic for Paediatrics and Adolescent Medicine, University Hospital Halle (Saale), Halle, Germany

${ }^{10}$ ImmunDefektCentrum Leipzig (IDCL), Klinikum St. Georg, Leipzig, Germany

${ }^{11}$ Pädiatrie 2, Olgahospital and Women's Clinic, Stuttgart, Germany

${ }^{12}$ Department of Paediatric Nephrology and Gastroenterology,

Universitätskinderklinik Wien, Wien, Austria

${ }^{13}$ Department of Pediatrics, University Hospital and Medical Faculty Carl Gustav Carus, Technische Universität Dresden, Dresden, Germany

Acknowledgements We are grateful to all patients and their parents for participating in the NRPD, BiKeR and the COVID-19 Survey of the DGPI and to all colleagues who contributed to this data collection. Dr Annett Lambrecht, Universitätskinderklinik Magdeburg; Professor Dr Johannes-Peter Haas, DZKJR Garmisch-Partenkirchen; Dr Ralf Trauzeddel, Helios-Klinikum Berlin-Buch; Dr Moritz Klaas, Vivantes Klinikum Berlin-Friedrichshain PD; Dr Jürgen Brunner, Universitätsklinderklinik Innsbruck; Dr Gonza Ngoumou, Universitätsmedizin Berlin—Charité; Dr Mareike Lieber, Universitätsmedizin Berlin—Charité; Dr Sae Lim von Stuckrad, Universitätsmedizin Berlin—Charité; Dr Lisa Lurz, Universitätsmedizin Berlin—Charité; Dr Peggy Rühmer, Helios Kliniken Vogtland; Dr Andrea Skrabl-Baumgartner, Universitätskinderklinik Graz; Professor Dr Tim Niehues, Helios Kliniken Krefeld PD; Dr Catharina Schütz, Universitätskinderklinik Dresden; Dr Anja Hauenherm, Städt. Kliniken St. Georg, Leipzig; Dr Friederike Blankenburg, Klinikum Stuttgart; Dr Anita Heinkele, Klinikum Stuttgart; Dr Frank Dressler, Medizinische Hochschule Hannover; Dr Tanja Hinze, Universitätskinderklinik Münster; Dr Sebastian Schua, St. Josef Stift Sendenhorst; Professor Dr Markus Hufnagel, Universitätskinderklinik Freiburg; Dr Melanie Römer, Kinderarztpraxis, Kempten; Dr Veit Grote, Dr von Hauner'sches Kinderspital, LMU München; Dr Regine Borchers, Universitätsklinikum Augsburg. We would like to thank Mrs Sabine Achtelstetter for her assistance with formatting the manuscript.

Contributors KM, MN, CS, AK and TK developed the study concept and design. $\mathrm{MN}$ was responsible for the administrative and technical support. The funding was obtained by KM, GH and JA. Data were acquired by KM, GH, MN, JK-D, CSi, RB, $H G, R H, M B, A H, W E, J A, A K$ and TK. Data were analysed and interpreted by SE, CS and KM. CS and SE drafted the manuscript, all other authors revised it critically for important intellectual content, agreed to be accountable for all aspects of the work and approved the final version of the manuscript. AK and TK are joint senior authors.

Funding The National Paediatric Rheumatological Database has been funded by AbbVie, Chugai, GSK and Novartis. The BiKeR has been supported by an unrestricted grant from Pfizer, Germany, AbbVie, Germany, Novartis, Germany and Roche, Germany. The COVID-19 Survey is supported by a grant from the Federal State of Saxony.

Disclaimer The funders had no influence on study design or on the collection, analysis or interpretation of the data, the writing of the manuscript or the decision to submit the manuscript for publication. Publication of the article was not contingent on approval by the study sponsors.

Competing interests KM has received honoraria for lectures/consulting from AbbVie, Pfizer, Novartis; GH has received grant/research support from AbbVie, Chugai, Roche, Novartis, Pfizer and MSD and speaker fees from AbbVie, Boehringer, Celgene, Chugai, GSK, MSD, Novartis, Pfizer, Roche and Sobi; AH: advisory boards: SOBI, Novartis.

Patient consent for publication Not required.

Ethics approval The NPRD was approved by the ethics committee of the Charité-Universitätsmedizin Berlin (EA1/044/07). The Biologics in Paediatric Rheumatology Registry (BiKeR) was approved by the ethics committee of the physician board Aerztekammer Nordrhein, Duesseldorf (reference number 2/2015/7441). The COVID-19 Survey was approved by the Ethics Committee of the Technische Universität (TU) Dresden (BO-EK-110032020).

Provenance and peer review Not commissioned; externally peer reviewed.

Data availability statement All data relevant to the study are included in the article or uploaded as supplemental information. Only de-identified participant data documented on specific questionnaires were used in this observational study.

Open access This is an open access article distributed in accordance with the Creative Commons Attribution Non Commercial (CC BY-NC 4.0) license, which permits others to distribute, remix, adapt, build upon this work non-commercially, and license their derivative works on different terms, provided the original work is properly cited, appropriate credit is given, any changes made indicated, and the use is non-commercial. See: http://creativecommons.org/licenses/by-nc/4.0/. 
ORCID iD

Claudia Sengler http://orcid.org/0000-0002-6703-0269

\section{REFERENCES}

1 Ding Y, Yan H, Guo W. Clinical characteristics of children with COVID-19: a meta-analysis. Front Pediatr 2020;8.

2 Tsabouri S, Makis A, Kosmeri C, et al. Risk factors for severity in children with coronavirus disease 2019: a comprehensive literature review. Pediatr Clin North Am 2021;68:321-38.

3 Minden K, Niewerth M, Listing J, et al. Health care provision in pediatric rheumatology in Germany--national rheumatologic database. J Rheumatol 2002;29:622-8.

4 Lawson-Tovey S, Strangfeld A, Hyrich KL, et al. EULAR COVID-19 registry: lessons learnt and future considerations. Ann Rheum Dis 2021. doi:10.1136/annrheumdis-2021-220319. [Epub ahead of print: 27 Apr 2021].

5 Gianfrancesco MA, Hyrich KL, Gossec L, et al. Rheumatic disease and COVID-19: initial data from the COVID-19 global rheumatology alliance provider registries. Lancet Rheumatol 2020;2:e250-3.

6 Horneff G, Schmeling H, Biedermann T, et al. The German etanercept registry for treatment of juvenile idiopathic arthritis. Ann Rheum Dis 2004;63:1638-44.

7 Dong Y, Mo X, Hu Y, et al. Epidemiology of COVID-19 among children in China. Pediatrics 2020;145:e20200702.

8 Götzinger F, Santiago-García B, Noguera-Julián A, et al. COVID-19 in children and adolescents in Europe: a multinational, multicentre cohort study. Lancet Child Adolesc Health 2020;4:653-61.

9 Mantovani A, Rinaldi E, Zusi C, et al. Coronavirus disease 2019 (COVID-19) in children and/or adolescents: a meta-analysis. Pediatr Res 2021;89:733-7.

10 King JA, Whitten TA, Bakal JA, et al. Symptoms associated with a positive result for a swab for SARS-CoV-2 infection among children in Alberta. CMAJ 2021;193:E1-9.

11 Hasseli R, Mueller-Ladner U, Schmeiser T, et al. National registry for patients with inflammatory rheumatic diseases (IRD) infected with SARS-CoV-2 in Germany (ReCoVery): a valuable mean to gain rapid and reliable knowledge of the clinical course of SARS-CoV-2 infections in patients with IRD. RMD Open 2020;6:e001332.

$12 \mathrm{Xu} \mathrm{X,} \mathrm{Han} \mathrm{M,} \mathrm{Li} \mathrm{T,} \mathrm{et} \mathrm{al.} \mathrm{Effective} \mathrm{treatment} \mathrm{of} \mathrm{severe}$ COVID-19 patients with tocilizumab. Proc Natl Acad Sci U S A 2020;117:10970-5.
13 Salama C, Han J, Yau L, et al. Tocilizumab in patients hospitalized with Covid-19 pneumonia. N Engl J Med 2021;384:20-30.

14 Chen Z, Hu J, Zhang Z. Efficacy of hydroxychloroquine in patients with COVID-19: results of a randomized clinical trial. medRxiv 2020.

15 Mahévas M, Tran V-T, Roumier M. No evidence of clinical efficacy of hydroxychloroquine in patients hospitalised for COVID-19 infection and requiring oxygen: results of a study using routinely collected data to emulate a target trial. medRxiv 2020

16 Kalil AC, Patterson TF, Mehta AK, et al. Baricitinib plus remdesivir for hospitalized adults with Covid-19. N Engl J Med 2021;384:795-807.

17 Koker O, Demirkan FG, Kayaalp G, et al. Does immunosuppressive treatment entail an additional risk for children with rheumatic diseases? A survey-based study in the era of COVID-19. Rheumatol Int 2020;40:1613-23.

18 Haberman R, Axelrad J, Chen A, et al. Covid-19 in immunemediated inflammatory diseases - case series from New York. $N$ Engl J Med 2020;383:85-8.

19 Jovani V, Calabuig I, Peral-Garrido ML, et al. Incidence of severe COVID-19 in a Spanish cohort of 1037 patients with rheumatic diseases treated with biologics and JAK-inhibitors. Ann Rheum Dis 2020:annrheumdis-2020-218152.

20 D'Silva KM, Serling-Boyd N, Wallwork R, et al. Clinical characteristics and outcomes of patients with coronavirus disease 2019 (COVID-19) and rheumatic disease: a comparative cohort study from a US 'hot spot'. Ann Rheum Dis 2020;79:1156-62.

21 Cordtz R, Lindhardsen J, Soussi BG, et al. Incidence and severeness of COVID-19 hospitalisation in patients with inflammatory rheumatic disease: a nationwide cohort study from Denmark. Rheumatology 2020. doi:10.1093/rheumatology/keaa897. [Epub ahead of print: 28 Dec 2020].

22 Gesundheit von Kindern und Jugendlichen in der Pandemie. Drucksache 19/28021. Berlin, 2021. Available: https://dserver bundestag.de/btd/19/283/1928348.pdf

23 Becker I, Horneff G. Risk of serious infection in juvenile idiopathic arthritis patients associated with tumor necrosis factor inhibitors and disease activity in the German biologics in pediatric rheumatology registry. Arthritis Care Res 2017;69:552-60.

24 Janda A, Schuetz C, Canna S, et al. Therapeutic approaches to pediatric COVID-19: an online survey of pediatric rheumatologists. Rheumatol Int 2021;41:911-20. 